\title{
Design of a Switching Flyback Transformer as High Voltage and Frequency Power Supply for Raw Milk Cold Plasma Sterilization
}

\author{
Muhamad Ibnu Fajar ${ }^{*}$, Elfahra Casanza Amalda ${ }^{2}$, Muhammad Ali Akram Syah ${ }^{3}$ \\ Nurussa'adah ${ }^{4}$ \\ ${ }^{1,4}$ Department of Electrical Engineering, Faculty of Engineering, \\ Universitas Brawijaya, Malang, East Java 65145, Indonesia. \\ ${ }^{2,3}$ Faculty of Veterinary Medicine, Universitas Brawijaya, Malang, East Java 65151, Indonesia \\ ${ }^{*}$ Corresponding author: \\ Email: muhamadibnu@student.ub.ac.id
}

\begin{abstract}
.
Plasma is one of the four fundamentals states of matter other than solid, liquid and gas. In terms of temperature, plasma can be classified into Hot Plasma occurs in thermal equilibrium, Thermal Plasma occurs in non-thermal equilibrium, Cold Plasma occurs in non-thermal equilibrium Cold plasma is an ultra-fast method of sterilization that operates under ambient temperatures (ideal for thermolabile products) at a low running cost and is environment-friendly. Therefore, technology is needed to solve the problem through the innovation of a milk sterilization machine with the principle of non-thermal plasma sterilization by utilizing plasma technology to reduce microbial contamination. The output frequency and voltage are determined through software simulation using voltmeter, oscilloscope, and counter timer. After 220VAC supplies a voltage step down and rectifier circuit to produce a 12VDC output voltage to supply the Switching circuit voltage and the Flyback Transformer driver. Rated voltage and frequency reach $20 \mathrm{kV} 15 \mathrm{kHz}$. The positive wire from the Flyback Transformer is connected to the acrylic box filled with water (positive electrode) and the ground wire is connected to another acrylic sheet (ground electrode. The milk medium to be sterilized can be placed on the positive electrode or between the ground and positive electrodes. Non-thermal plasma has an effective ability to decontaminate bacteria. This is because plasma is composed of a mixture of ionized particles, reactive radical molecules, and also ultraviolet Schematic circuit design has been successfully simulated. Acrylic sterilization media designs can be used to generate plasma. The mechanism of bacterial sterilization in milk is by the activity of inhibiting bacterial biofilms which can lead to bacterial DNA damage through reactive species in the non-thermal plasma used. Milk quality testing is carried out based on comparison of supporting literature with milk quality requirements according to SNI 3950: 2014.
\end{abstract}

Keywords: Cold plasma, corona discharge, flyback transformer, milk, sterilization.

\section{INTRODUCTION}

Plasma is defined as an ionized gas. This concept was first described in 1928 by Langmir and Tonks [1]. Plasma is one of the four fundamentals states of matter other than solid, liquid and gas. The structural form of plasma are filaments, beams and double layers of beams because of the influence of the electromagnetic field [2]. In http://ijstm.inarah.co.id 
terms of temperature, plasma can be classified into three, namely Hot Plasma which occurs in a state of thermal equilibrium which has a temperature of more than $10^{6} \mathrm{~K}$, Thermal Plasma occurs in a non-thermal equilibrium state having a temperature of $3000-10^{5} \mathrm{~K}$, Cold Plasma occurs in a non-thermal equilibrium state between the temperature of electrons and gases has a temperature equal to room temperature [3]. In general, high-voltage and high-frequency generating circuits require a complicated circuit scheme, but as research develops, one simple generation method is obtained, namely through a flyback transformer [4].

Cold plasma consists of gas molecules with moderate temperatures and electrons with higher temperatures. In contrast, the TP consists of electrons and gas molecules with several thousands of degrees Kelvin temperature, and these species are found in equilibrium [5]. Cold plasma techniques have attracted food scientists and researchers and recently been studied for potential inactivation of microorganisms [6]. Cold plasma is an ultra fast method of sterilization that operates under ambient temperatures (ideal for thermolabile products) at a low running cost and is environment-friendly. There are various plasma techniques related to the method of generation [7]. Dielectric barrier discharge (DBD) plasma is one of these techniques. It has been used to sterilize raw milk however, anaerobic bacteria have been surviving after DBD plasma treatment [8].

Therefore, technology is needed to solve the problem through the innovation of a milk sterilization machine with the principle of non-thermal plasma sterilization by utilizing plasma technology to reduce microbial contamination. The sterilized milk will immediately end up in a closed container that is protected from external contamination so that it can minimize microbial contamination. This innovation also supports programs to increase milk production in Indonesia in line with increasing interest in consumption that is not matched by milk production. In addition, this tool is expected to reduce the number of imports of milk and encourage a program for Indonesia's food independence.

\section{METHODS}

In the implementation of this research using simulation software to determine the output parameters in the form of voltage and frequency. The system consists of an electric circuit and a cold plasma generating medium for sterilization. The electrical circuit consists of a Power Supply and a Switching Flyback Transformer. Plasma generator media made of acrylic arranged and connected to the positive wire and ground of the Flyback Transformer. The hardware system design is shown in Figure 1. 


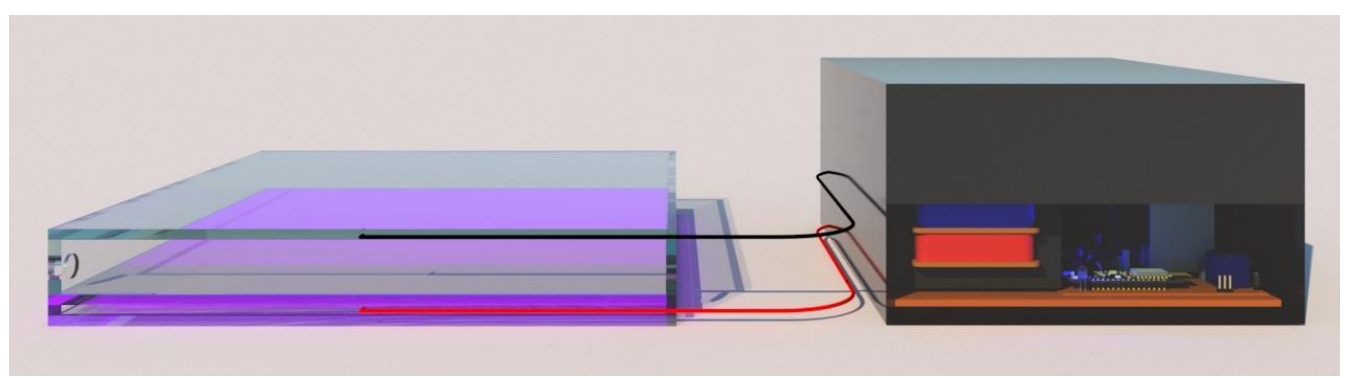

Fig. 1. Hardware System Design

The Flyback Transformer driver circuit schematic as shown in Figure 2. The switching circuit consists of a pair of Zener Diodes and MOSFETs which are used to increase the input voltage to a high frequency output voltage which drives a Flyback Transformer.

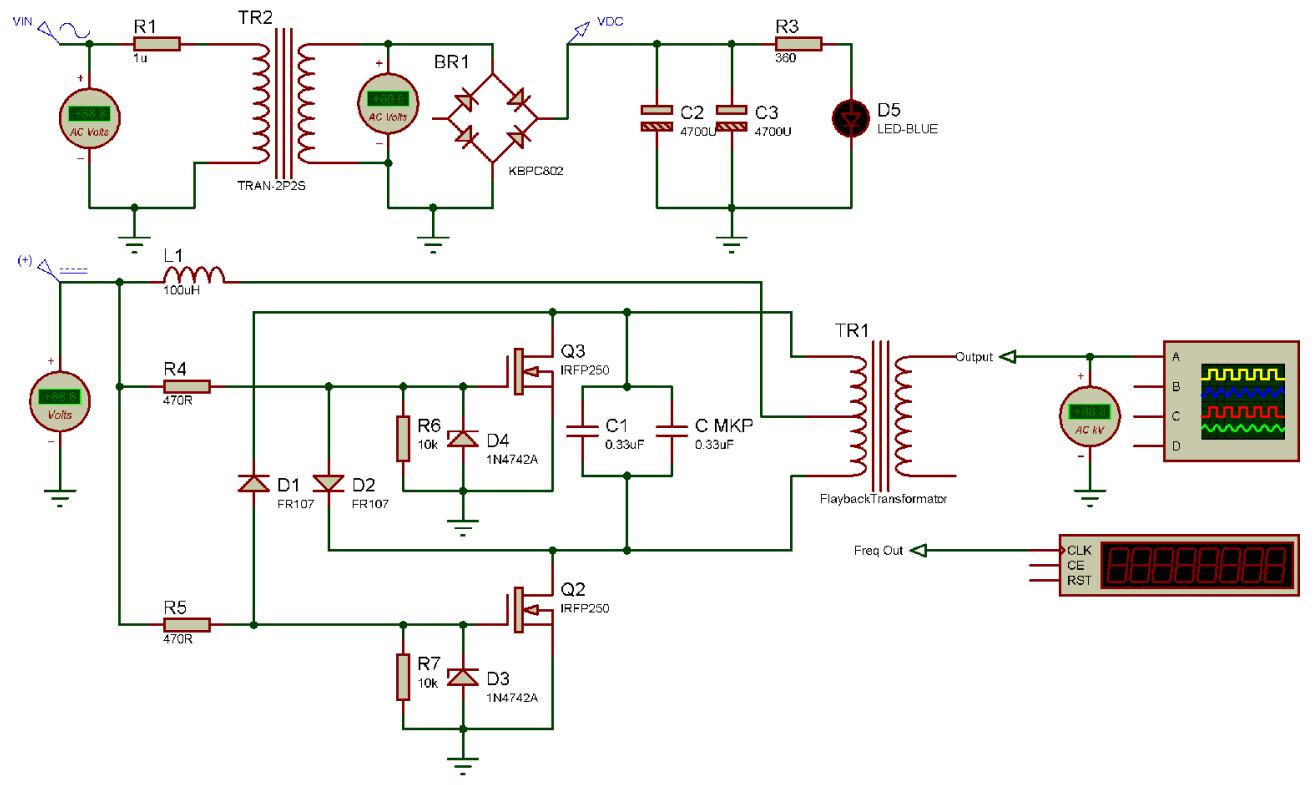

Fig. 2. Schematic Circuit Flyback Transformer Driver

In the corona discharge and dielectric barrier discharge, ozone is the main reaction product, whereas in the other plasmas, oxygen atoms represent a large fraction of the reactive species. In a low-pressure glow discharge the concentrations of ions and atoms are lower than in an atmospheric-pressure plasma. However, the impingement rate of these species on a substrate may be about the same in both cases, since the flux to the surface increases with decreasing pressure. Taking into account all the properties of the plasmas, it appears that the atmospheric-pressure plasma jet exhibits the greatest similarity to a low-pressure glow discharge. Consequently, this device shows promise for being used in a number of materials applications that are now limited to vacuum [2]. 


\section{RESULT AND DISCUSSION}

The output frequency and voltage are determined through software simulation using a voltmeter, oscilloscope, and a counter timer. Figure 3 shows the Output of the circuit at the secondary coil of Flyback Transformer. After 220V AC supplies a voltage step down and rectifier circuit to produce a $12 \mathrm{~V}$ DC output voltage to supply the Switching circuit voltage and the Flyback Transformer driver. Rated voltage and frequency reach $20 \mathrm{kV} 15 \mathrm{kHz}$.

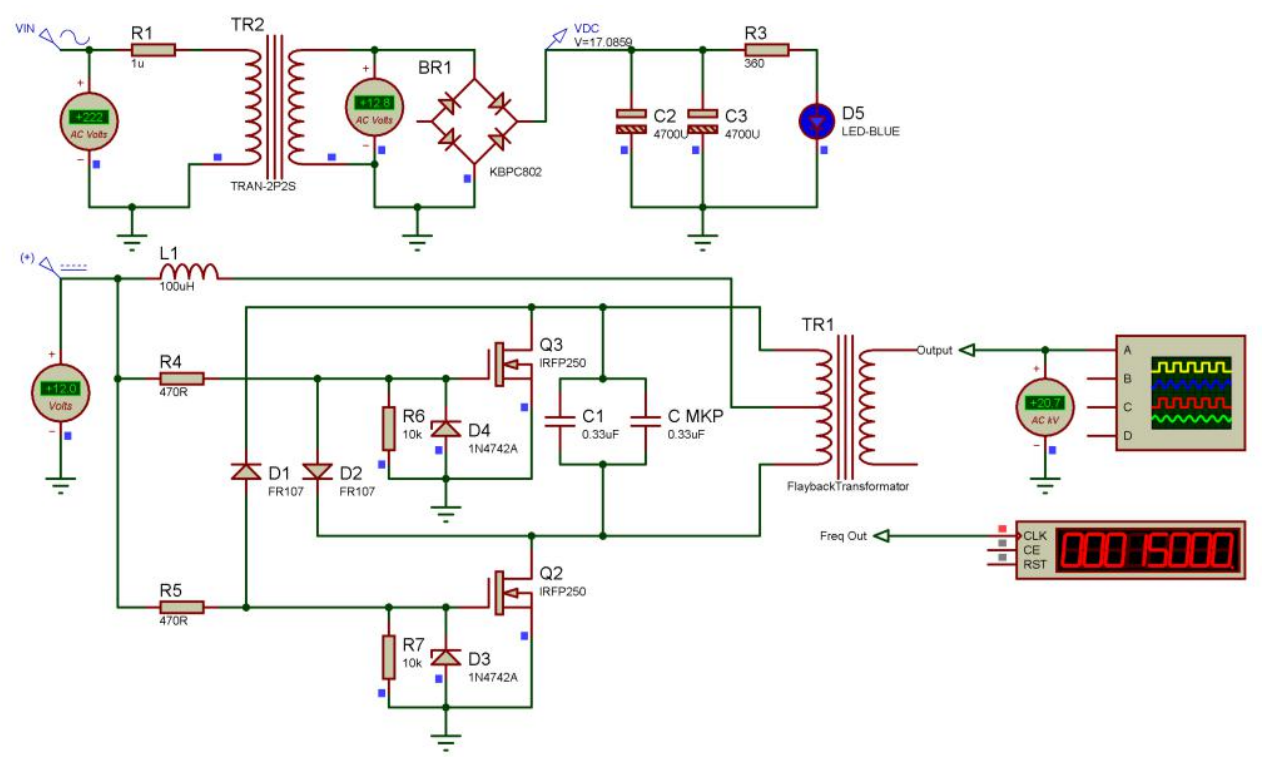

Fig. 3. Software Simulation Electric Circuit

Flyback transformer has some internal parts i.e. a secondary winding, a primary winding, and the auxiliary windings. The secondary winding has many turns. This winding, as well as the whole flyback body, is supported by the HV (High Voltage) isolation so that it can withstand an HV up to tens of kilovolts. The primary winding is connected to the converter circuit. The auxiliary windings which are to generate specific voltage levels.

In stage 1, the capacitor is completely discharged as the drain voltages approach zero. During this stage, all resonant energy is stored in the inductor which is the primary winding of the flyback transformer. Because there is no voltage, thus there is no power transfer from the primary winding to the secondary winding as well as the output voltage. The cross-coupling circuit, which is provided by the diodes D3 and D4, ensures only one MOSFET will turn ON as another turn OFF. When the Q2 turns OFF, and Q3 turns $\mathrm{ON}$, the resonance between the primary winding and the capacitor occurs which is the beginning of stage 2 .

Stage 2, the energy which was stored in the primary winding is transferred as a current to the capacitor and charges it. During the charging process, the drain voltage 
of Q2 rises simultaneously. When the entirety energy in the primary winding has completely transferred to the capacitor, the charging process stops. As the charging process stops, the capacitor undergoes the discharging process so that the current flows back to the primary winding, followed by the falling of the Q2 drain voltage. During this stage, the energy is transferred from the primary winding to the secondary winding and the current flows through the diode to the load while charging the capacitor.

Stage 3 begins after the completion of the capacitor discharging process. As the discharging process completed, the primary winding stores all energy just like the stage 1 but in opposite voltage polarity. The MOSFET Q2 begins to turn ON while Q3 turns OFF. No power transfer from the primary winding to the secondary winding occurs at this stage. Stage 4 has a similar process with the stage 2 [9].

The positive wire from the Flyback Transformer is connected to the acrylic box filled with water (positive electrode) and the ground wire from the Flyback Transformer is connected to another acrylic sheet (ground electrode) which is on the other side. The milk medium to be sterilized can be placed on the positive electrode or between the ground and positive electrodes.

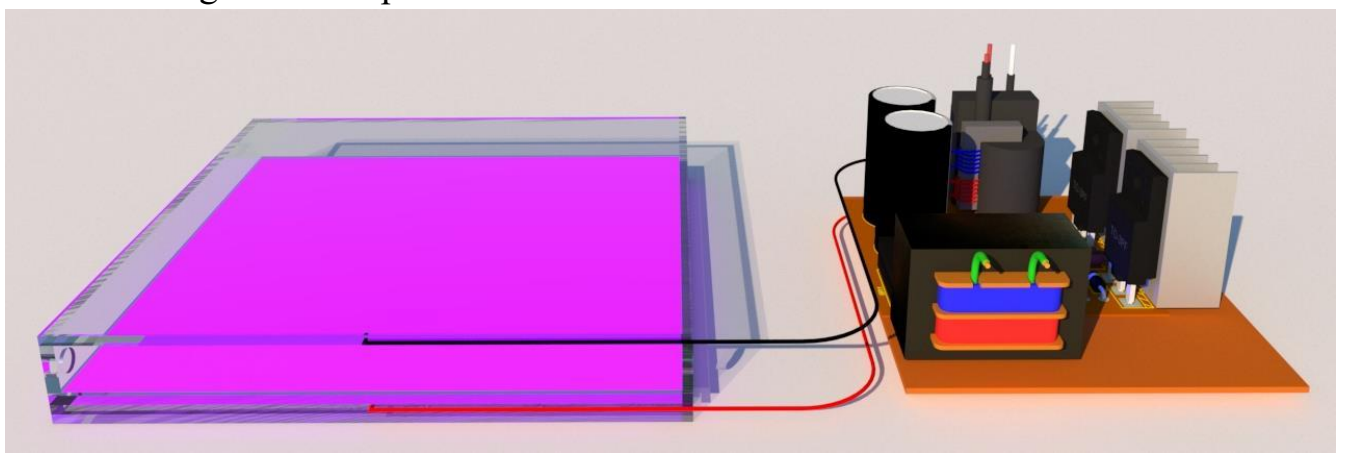

Fig. 4. Milk Cold Plasma Sterilization Circuit

Non-thermal plasma has an effective ability to decontaminate bacteria. This is because plasma is composed of a mixture of ionized particles, reactive radical molecules, and also ultraviolet (UV) [10]. One of the molecules emitted by ultraviolet is NO (Nitrogen Oxide) [11]. NO is one of the main agents of bacterial inactivation because it can destroy cells by dimerizing the thymine base in the DNA strands, which results in disruption of the DNA replication process. Apart from Nitrogen Oxide, nonthermal plasma also produces several other reactive molecules such as $\mathrm{OH}, \mathrm{O}_{2}$ and $\mathrm{O}_{3}$. These molecules are known to also play a role in the inactivation process of bacteria. Hydroxyl radical $(\mathrm{OH})$ is a type of reactive oxygen that causes death in bacteria through an oxidative process. Hydroxyl radicals can also kill bacteria by destroying unsaturated fatty acids on cell membranes [11] $\mathrm{O}_{2}$ - helps the formation of more reactive radical molecules, whereas $\mathrm{O}_{3}$ can interfere with cellular respiration [11] The protein molecules that make up the bacterial cell membrane are known to be susceptible to oxidation processes so that giving non-thermal plasma treatment to bacteria can cause erosion which results in the breaking of the bacterial cell wall. In 
addition to causing cell wall rupture, the use of non-thermal plasma can also inhibit the formation and destruction of biofilms through the oxidation process by reactive molecules on the surface of hydrophibicity [12]. Milk quality testing is carried out by conducting literature studies and looking for secondary data from relevant previous studies. Standardization of milk refers to SNI 3950: 2014 regarding Ultra High Temperature Milk and SNI 3141.1: 2011 concerning Fresh Cow Milk.
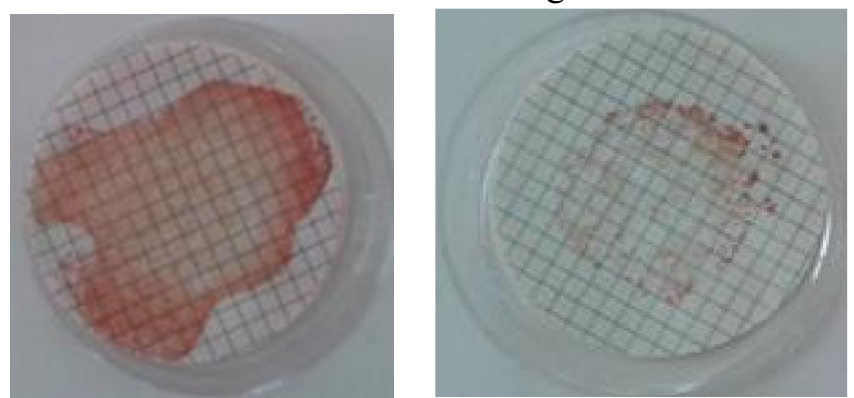

Fig. 4. The Differences of Bacterial Clearance Between (a) Untreated Plasma and (b) Treated 3kv Voltage and 3 Mins Applied. [5]

The experimental study shows that the effect of DBD plasma application has potentially reduced the number of bacteria in agar plate. The study designed in changing time, voltage and frecuency parameters, therefore found the optimal results were achieved at $500 \mathrm{~Hz}$ fixed frecuency with $3 \mathrm{kV}$ voltage in 3 mins time interval applied [5].

\section{CONCLUSION}

Schematic circuit design for the plasma generator consisting of the switching, driver and Flyback Transformer have been successfully simulated. Acrylic sterilization media designs can be used to generate plasma. The mechanism of bacterial sterilization in milk is by the activity of inhibiting bacterial biofilms which can lead to bacterial DNA damage through reactive species in the non-thermal plasma used. Milk quality testing is carried out based on comparison of supporting literature with milk quality requirements according to SNI 3950: 2014 showing that several aspects such as the number of bacterial colonies do not reach the maximum threshold

\section{ACKNOWLEDGMENTS}

The authors are grateful to RistekDikti and Brawijaya University for supporting this research program and providing any facilities needed.

\section{REFERENCES}

[1] D. Kind, Pengantar Teknik Ekspremental Tegangan Tinggi, ITB, 1993.

[2] A. Schutze, J. Y. Jeong, S. E. Babayan, J. Park, G. S. Selwyn and R. F. Hicks, Atmospheric-Pressure Plasma Jet: A Review and Comparison to Other Plasma Sources, IEEE Transactions on Plasma Science, 26:6, 1998, pp. 1685-1694. 
[3] M. Nur, Fisika Plasma dan Aplikasinya, Universitas Diponegoro, 2011, pp. 15-26

[4] N. Barsoum, G. I. Stanley, Design of High Voltage Low Power Supply Device, Universal Journal of Electrical \& Electronic Engineering, 3:1, 2015, pp. 6-12.

[5] Yakup, The Effect of Dielectric Barrier Discharge Plasma Treatment on the Microorganisms Found in Raw Cow's Milk, Turkish Journal of Agricultural Research, 3:2, 2016, pp. 169-173.

[6] N. N. Misra, B. K. Tiwari, K. S. M. S. Rahavarao, P. J. Cullen, Nonthermal Plasma Inactivation of Foodborne Pathogens, Food Engineering Reviews, 3, 2011, pp. 3-4.

[7] S. M. Banu, P. Sasikala, D. Aruna, V. Kavitha, G. Yazhini, R. Lavanya, Cold Plasma as A Novel Food Processing Technology, International Journal of Emerging Trends in Engineering and Development, 4:2, 2012, pp. 803-818

[8] H. J. Kim, H. I. Yong, S. Park, K. Kim, W. Choe, C. Jo, Microbial Safety and Quality Attributes of Milk Following Treatment with Atmospheric Pressure Encapsulated Dielectric Barrier Discharge Plasma, Food Control, 47, 2015, pp. 451-456.

[9] S. L. McClusky, High Voltage Resonant Self-Tracking Current-Fed Converter, California Polytechnic State University, 2010.

[10] Sakudo, Akikazu, Y. Yagyu, T. Onodera, Disinfection and Sterilization Using Plasma Technology: Fundamentals and Future Perspectives for Biological Applications, International Journal of Molecular Sciences, 20, 2019, p.20

[11] Tian, Ye, P. Sun, H. Wu, N. Bai, R. Wang, W. Zhu, J. Zhang F. Liu. Inactivation of Staphylococcus Aureus and Enterococcus Faecalis by a Direct-Current, Cold Atmospheric-Pressure Air Plasma Microjet, Journal of Biomedical Research, 24:4, 2010, pp.264-269.

[12] Fallon, Muireann, M. Boyle, S. Kennedy, S. Daniels, H. Humphreys, Cold Atmospheric Plasma, the Removal of Blood from Steel and Its Effect on Staphylococcal Biofilm Formation. A Pilot Study, Clinical Plasma Medicine, 2020, pp. 19-20 